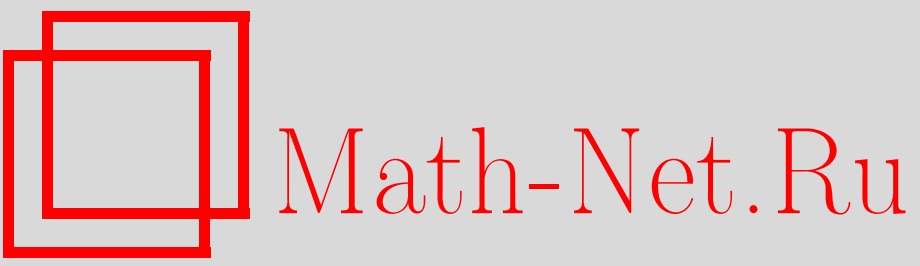

М. Л. Бланк, Точный анализ динамических систем, возникающих в моделях транспортных потоков, УМН, 2000, том 55, выпуск 3, 167-168

DOI: https://doi.org/10.4213/rm295

Использование Общероссийского математического портала Math-Net.Ru подразумевает, что вы прочитали и согласны с пользовательским соглашением

http://www . mathnet.ru/rus/agreement

Параметры загрузки:

IP : 54.209 .52 .79

26 апреля 2023 г., 03:24:51 


\title{
ТОЧНЫЙ АНАЛИЗ ДИНАМИЧЕСКИХ СИСТЕМ, ВОЗНИКАЮЩИХ В МОДЕЛЯХ ТРАНСПОРТНЫХ ПОТОКОВ
}

\author{
М. Л. БЛАнК
}

При помощи техники двойственных отображений получены точные результаты динамики нескольких моделей транспортных потоков, для которых также получено полное описание их предельных режимов в пространстве конфигураций. Используя эти результаты, мы изучаем движение пассивной быстрой частищы, двигающейся по потоку/против потока медленных частиц.

1. Поводом для настоящей работы послужило следующее наблюдение: в медленно двигающейся толпе быстрее идти против общего движения, чем по нему. Этот эффект особенно заметен при движении около границы двух медленных “потоков" людей, двигающихся в противоположных направлениях (например, в переходе метро). Очевидно, это наблюдение противоречит стандартной модели диффузии частищы по потоку/против потока и, как минимум, говорит об очень специальной (неслучайной) структуре транспортных потоков.

Начнем с простейшей одномерной модели транспортного потока, введенной в [1]. Эта модель, которую мы будем называть моделью с медленными частицами, описывается следующей динамической системой с дискретным временем и дискретным фазовым пространством - решеткой, на которой расположены частицы. В следующий момент времени каждая частица либо передвигается вперед на одну позицию - если она свободна, либо остается на месте - в противном случае. В случае конечной решетки с периодическими граничными условиями анализу (в основном численному) этой модели и некоторых ее обобщений в последнее время было посвящено большое число публикаций (см. [1]-[6] и дальнейшие ссылки в них). Наиболее интересным явлением, обнаруженным в этих работах, является нетривиальная зависимость $\mathrm{V}(\rho)$ средней скорости движения частиц от их плотности, равная 1 при $\rho \in[0,1 / 2]$ и $\frac{1}{\rho}-1$ при $\rho \in(1 / 2,1]$. Ниже мы выведем этот результат при помощи техники двойственных отображений для произвольных конечных и бесконечных решеток и начальных конфигураций. Кроме того, мы дадим полное описание предельных множеств (соответствующих стационарным транспортным потокам) и точную оценку длины переходного периода.

С точки зрения теории динамических систем описанная выше модель может быть представлена следуюшим образом. Пусть $\mathbf{X}=\{0,1\}^{\mathbb{Z}^{1}}$ - множество всех возможных конфигураций - бинарных последовательностей $X$, единицы в которых соответствуют частищам, а нули - незанятым позициям на решетке. Рассмотрим отображение $T: \mathbf{X} \rightarrow \mathbf{X}$,

$$
T X(x):= \begin{cases}1, & \text { если } X(x)=0 \text { и } X(x-1)=1 \text { или } X(x)=X(x+1)=1, \\ 0 & \text { в остальных случаях. }\end{cases}
$$

Группу из $k$ последовательно стоящих частищ, $k>1$, мы назовем кластером; а частицу в позиции $x$ (т.е. $X(x)=1)$, позиция после которой не занята (т.е. $X(x+1)=0)$, назовем свободной. Будем называть конфигурацию $X \in \mathbf{X}$ регулярной, если имеется число $\rho=\rho(X)$ (плотность частиц) и монотонная функция $\varphi(N), \varphi(N) \rightarrow 0$ при $N \rightarrow \infty$, такие, что для любого $N$ число частиц с координатами от $n+1$ до $n+N$ отличается от $N \rho$ не более чем на $N \varphi(N)$ для любого $n$. Заметим, что конфигурация на конечной решетке длины $n$ с периодическими граничными условиями соответствуют $n$-периодической конфигурации на бесконечной решетке, которая удовлетворяет условию регулярности с $\varphi(N)=n \rho(1-\rho) / N$. Под средней (по пространству) скоростью (частиц) $\mathrm{V}(X)$ понимается среднее значение (если оно корректно определено) перемещения частиц в конфигурации $X$ во время следующей итерации отображения $T$.

Теорема 1. Для любой регулярной начальной конфигурации $X \in \mathbf{X}$ с плотностью $\rho=1 / 2$ через не более чем $t_{c}(X)=\frac{1}{2} \varphi^{-1}\left(\left|\frac{1}{2}-\rho(X)\right|\right)$ итераций отображения $T$ средняя скорость станет равна $\mathrm{V}=\min \left(1, \frac{1}{\rho}-1\right)$, и при любом $t \geqslant t_{c}(X)$ справедлива следующая альтернатива: конфигурация $T^{t} X$ состоит либо только из свободных частиц, либо не имеет кластеров незанятых позиций. Более того, при любом $n$ для $n$-периодических начальных конфигурачий ограничение $\rho \neq 1 / 2$ снимается, для $t_{c}(X)$ справедлива

Работа частично поддержана грантами РФФИ и INTAS. 
лучшая оценка $t_{c}(X)=\min (\rho(X) N, N-\rho(X) N)$ и при $t \geqslant t_{c}(X)$ последовательность $\left\{T^{t} X\right\}_{t}$ становится $n$-периодической по $t$.

ДокАЗАТЕЛЬСТВО этой теоремы и ряда других результатов настоящей работы основано на идее введения двойственной динамической системы $\left(T^{*}, \mathbf{X}^{*}\right)$, описывающей динамику незанятых позиций на решетке под действием основного отображения $T$. Здесь для конфигурации $X \in \mathbf{X}$ двойственная конфигурация $X^{*}$ определяется соотношением $X^{*}(x)=1-X(x)$ для всех $x$. Можно показать, что $(T X)^{*}=T^{*} X^{*}$ при всех $X \in \mathbf{X}$. Для рассматриваемой модели отображение $T^{*}$ отличается от $T$ только направлением движения частищ, что сводит анализ к конфигурациям низкой плотности $\rho \in[0,1 / 2]$, поскольку бо́льшая плотность соответствует плотности незанятых позиций, меньшей $1 / 2$. Это наблюдение резко упрощает задачу, поскольку динамика в случае высокой плотности частиц нетривиальна и трудно поддается непосредственному анализу. Далее, показывая, что длина любого кластера частиц не может возрастать (т.е. в этой модели не могут возникать транспортные пробки), а число свободных частиц убывать, мы приходим к описанной в формулировке теоремы альтернативе, что и приводит к требуемым оценкам.

2. Рассмотрим теперь модель движения с быстрыми частицами, отличающуюся от предыдущей тем, что на каждом шагу частица сдвигается вперед до следующей занятой позиции.

Теорема 2. Для любой начальной конфигурации $X$, удовлетворяющей закону больиих чисел с плотностью $\rho(X) \notin\{0,1\}$, средняя скорость частиц не зависит от времени и равна $\frac{1}{\rho(X)}-1$.

Качественно динамика этой модели богаче, чем в модели с медленными частицами, например, транспортные пробки типичны даже для конфигураций малой плотности. С другой стороны, несмотря на это средняя скорость движения частиц $\mathrm{V}(\rho)$ для этой модели совпадает с предыдущим случаем при высокой плотности и аналитически продолжает ее при малой плотности.

3. Дадим теперь математическое описание наблюдения о движении пассивной быстрой частицы (имитирующей поведение спешащего прохожего) в медленном транспортном потоке, которое мы сформулировали в начале заметки. Упрощая ситуацию, мы будем полагать (как обычно делают в гидродинамике), что движение нашей быстрой частицы не влияет на транспортный поток и описывается следующим образом. Положим $\tau_{X}^{+}(y):=\min (x: y<x$ и $X(x)=1)$, $\tau_{X}^{-}(y):=\max (x: y>x$ и $X(x)=1)$. Тогда совместная динамика конфигурации медленных частиц $X \in \mathbf{X}$ и положения быстрой частицы $y \in \mathbb{Z}^{1}$ определяется косым произведением отображений $T$ и одного из $\tau^{ \pm}$(знак соответствует движению по потоку/против потока), т.е. $\mathscr{T}_{ \pm}(X, y):=\left(T X, \tau_{X}^{ \pm}(y)\right)$. Под скоростью в момент $t$ пассивной частицы будем понимать суммарное расстояние (со знаком), пройденное ею к этому моменту времени, деленное на $t$. Опираясь на полученное полное описание предельных множеств модели медленных частиц, мы получаем следующий результат.

ТЕОРема 3. Для любой регулярной начальной конфигурации с плотностью $\rho(X) \notin$ $\{0,1 / 2,1\}$ в случае неограниченной решетки средняя скорость быстрой частицы стремится (по t) $к 1$ при $\rho \leqslant 1 / 2$ и движении вперед (по потоку) и $к-\max \left(1, \frac{1}{\rho(X)}-1\right)$ при движении назад (против потока).

\section{СПИСОК ЛИТЕРАТУРЫ}

[1] Nagel K., Schreckenberg M. // J. Physique I. 1992. V. 2. P. 2221-2229. [2] Fukś H. // Phys. Rev. E. 1999. V. 60. P. 197-202, comp-gas/9902001. [3] Krug J., Spohn H. // Phys. Rev. A. 1988. V. 38. P. 4271-4283. [4] Nagel K., Herrmann H. J. // Phys. A. 1993. V. 199. P. 254-269. [5] Boccara N., Fukś H. Critical behavior of a cellular automaton highway traffic model // adap-org/9705003. [6] Simon P. M., Nagel K. // Phys. Rev. E. 1998. V. 58. P. 1286-1295, cond-mat/9801022. 